\title{
COMPLEMENTARY VARIATIONAL PRINCIPLES FOR LARGE DEFLECTIONS OF A CANTILEVER BEAM*
}

\author{
$\mathrm{By}$ \\ R. I. REEVES \\ University of York, England
}

\begin{abstract}
Recent results on complementary variational principles and error bounds are applied to two problems concerning the large deflection of a horizontal cantilever. The results are illustrated by obtaining accurate variational solutions in the form of simple polynomials.

1. Introduction. Complementary variational principles have recently been devel$[1,2]$ for a wide class of linear and nonlinear boundary-value problems including many boundary conditions. In certain problems the basic action functional represents a physical quantity of interest such as energy or capacity, and these principles provide upper and lower bounds for these quantities. In nonlinear problems, when attention is centered upon the boundary-value problem itself, complementary variational principles provide the basis for the construction of approximate solutions and error bounds.

The purpose of this paper is to present complementary variational principles relevant to problems occurring in the large-deflection theory of flexible bars. The results are illustrated by two cases of a horizontal cantilever subjected to a vertical load. For these problems it is possible to transform the differential equation and boundary conditions into a fairly simple integral equation and from these representations two sets of complementary variational principles are obtained. The integral equation approach produces the better lower bound of the action functional while using fewer variational parameters. This fact is used in an error bound for the approximate solution.
\end{abstract}

2. The class of problems. We consider boundary-value problems with equations of the form

$$
L \phi=T^{*} T \phi=f(\phi) \text { in } V
$$

and boundary conditions

$$
\sigma_{T} \phi=0 \text { on } \partial V_{1}, \quad \sigma_{T}^{*} T \phi=0 \text { on } \partial V_{2} .
$$

Here $L$ is a selfadjoint positive definite operator (see Mikhlin [3]), possibly a differential, integral or matrix operator, $V$ is some region of space and $\partial V_{1}+\partial V_{2}=\partial V$ makes up the boundary of $V$.

* Received February 20, 1974. The author thanks Drs. A. M. Arthurs and N. Anderson for their help in the preparation of this paper and the Science Research Council for the award of a Research Studentship. 
The linear operator $T$ and its adjoint $T^{*}$ satisfy the equation

$$
\int_{V} U T \Phi d V=\int_{V}\left(T^{*} U\right) \Phi d V+\int_{\partial V} U \sigma_{T} \Phi d B
$$

for all admissible $U, \Phi$, where $\sigma_{T}$ is a linear operator restricted to $\partial V$, having an adjoint $\sigma_{T}{ }^{*}$ defined by

$$
U \sigma_{T} \Phi=\left(\sigma_{T}{ }^{*} U\right) \Phi \quad \text { on } \partial V .
$$

For an example of the operators $T, T^{*}$ and $\sigma_{T}$ see Sec. 7 .

To introduce variational principles associated with Eqs. (2.1)-(2.3) we rewrite them in the canonical form

$$
\begin{aligned}
T \phi & =u=\partial H / \partial u \text { in } \quad V, \\
T^{*} u & =f(\phi)=\partial H / \partial \phi \text { in } \quad V
\end{aligned}
$$

with

$$
\begin{array}{rll}
\sigma_{T} \phi=0 & \text { on } & \partial V_{1}, \\
\sigma_{T}{ }^{*} u=0 & \text { on } & \partial V_{2} .
\end{array}
$$

A suitable Hamiltonian $H$ in Eqs. (2.6), (2.7) is

$$
H(u, \phi)=\frac{1}{2} u^{2}+F(\phi)
$$

where

$$
F(\phi)=\int^{\phi} f(\psi) d \psi
$$

3. Variational principles. Following Arthurs $[1,2]$ we introduce the action functional $I(U, \Phi)$ of the form

$$
\begin{aligned}
I(U, \Phi) & =\int_{V}\{U T \Phi-H(U, \Phi)\} d V-\int_{\partial V_{1}} U \sigma_{T} \Phi d B \\
& =\int_{V}\left\{\left(T^{*} U\right) \Phi-H(U, \Phi)\right\} d V+\int_{\partial V_{2}}\left(\sigma_{T}{ }^{*} U\right) \Phi d B
\end{aligned}
$$

by Eqs. (2.4), (2.5), and $H(U, \Phi)$ is defined by Eq. (2.10).

It then follows that

(a) First variational principle: For arbitrary independent functions $U$, $\Phi$ the functional $I(U, \Phi)$ is stationary at $(u, \phi)$, the solution pair of the boundary-value problem described in Eqs. (2.6)-(2.9).

We denote this value of the functional by

$$
I(u, \phi)=I(\phi)=\int_{V}\left\{\frac{1}{2} \phi f(\phi)-F(\phi)\right\} d V .
$$

(b) Second variational principle: Using Eq. (3.1) we define a functional $J\left(\Phi_{1}\right)$ by

$$
J\left(\Phi_{1}\right)=I\left(U\left(\Phi_{1}\right), \Phi_{1}\right), \quad U\left(\Phi_{1}\right)=T \Phi_{1}
$$

where $\Phi_{1}$ is any admissible function which satisfies the condition 


$$
\sigma_{T} \Phi_{1}=0 \text { on } \partial V_{1}
$$

Then we see that

$$
\begin{aligned}
J\left(\Phi_{1}\right) & =\int_{V}\left\{\frac{1}{2}\left(T \Phi_{1}\right)^{2}-F\left(\Phi_{1}\right)\right\} d V \\
& =\int_{V}\left\{\frac{1}{2} \Phi_{1} L \Phi_{1}-F\left(\Phi_{1}\right)\right\} d V+\frac{1}{2} \int_{\partial V_{2}}\left(\sigma_{T} * T \Phi_{1}\right) \Phi_{1} d B
\end{aligned}
$$

using Eqs. (2.4), (2.5) and (3.5).

Also

$$
J\left(\Phi_{1}\right)=I(\phi)+\Delta J
$$

where

$$
\Delta J=\frac{1}{2} \int_{V}\left\{\left(T\left(\Phi_{1}-\phi\right)\right)^{2}-\left(\Phi_{1}-\phi\right)^{2} \frac{\overline{d f}}{d \phi}\right\} d V,
$$

the bar over the derivative indicating that it is to be evaluated for some function $\phi+$ $\eta\left(\Phi_{1}-\phi\right), 0<\eta<1$. Eq. (3.8) shows that $J\left(\Phi_{1}\right)$ is stationary at $\phi$ and further if

$$
-(d f / d \psi)(\psi) \geq 0 \text { in } V \text { for all } \psi
$$

we see from Eq. (3.9) that

$$
\Delta J \geq 0 \text { for all } \Phi_{1}
$$

and so from (3.8) we have the global minimum principle

$$
I(\phi) \leq J\left(\Phi_{1}\right)
$$

for all admissible functions $\Phi_{1}$ satisfying condition (3.5).

(c) Third variational principle: Using Eq. (3.2) we define a functional $G(U)$ as follows:

$$
G(U)=I(U, \Phi(U)), \quad \Phi(U)=f^{-1}\left(T^{*} U\right) \text { in } V
$$

where $U$ is an admissible function satisfying the condition

$$
\sigma_{T}^{*} U=0 \text { on } \partial V_{2} \text {. }
$$

Then we see that

$$
\begin{aligned}
G(U) & =\int_{V}\left\{\left(T^{*} U\right) f^{-1}\left(T^{*} U\right)-\frac{1}{2} U^{2}-F\left[f^{-1}\left(T^{*} U\right)\right]\right\} d V \\
& =I(\phi)+\Delta G
\end{aligned}
$$

where

$$
\Delta G=-\frac{1}{2} \int_{V}\left\{(U-u)^{2}-\left(f^{-1}\left(T^{*} U\right)-\phi\right)^{2} \frac{\overline{g f}}{d \phi}\right\} d V,
$$

the bar over the derivative again denoting that it is to be evaluated at some function $u+\eta(U-u), 0<\eta<1$. Eq. (3.16) shows that $G(U)$ is stationary at $u$ and further if

$$
-(d f / d \psi)(\psi) \geq 0 \text { in } V \text { for all } \psi
$$


we see from (3.17) that

$$
\Delta G \leq 0 \text { for all } U,
$$

and so by equation (3.16) we have the global maximum principle

$$
G(U) \leq I(\phi)
$$

for all admissible functions $U$ satisfying condition (3.14).

Since the functions $u$ and $\phi$ are related by $u=T \phi$ in Eq. (2.6), we consider $U$ to have the form $U=T \phi_{2}$, where $\Phi_{2}$ is an approximation to $\phi$. Substituting in (3.15) and changing notation slightly by writing $G\left(\Phi_{2}\right)$ for $G\left(T \Phi_{2}\right)$, we have

$$
\begin{aligned}
G\left(\Phi_{2}\right) & =\int_{V}\left\{\left(T^{*} T \Phi_{2}\right) f^{-1}\left(T^{*} T \Phi_{2}\right)-\frac{1}{2}\left(T \Phi_{2}\right)^{2}-F\left[f^{-1}\left(T^{*} T \Phi_{2}\right)\right]\right\} d V \\
& =\int_{V}\left\{\left(L \Phi_{2}\right)\left[f^{-1}\left(L \Phi_{2}\right)-\frac{1}{2} \Phi_{2}\right]-F\left[f^{-1}\left(L \Phi_{2}\right)\right]\right\} d V-\frac{1}{2} \int_{\partial V_{1}} T \Phi_{2}\left(\sigma_{T} \Phi_{2}\right) d B
\end{aligned}
$$

where Eqs. (2.4), (2.5) and condition (3.14) have been used.

By Eq. (3.19) we see

$$
G\left(\Phi_{2}\right) \leq I(\phi)
$$

for all functions $\Phi_{2}$ satisfying the condition

$$
\sigma_{T}^{*} T \Phi_{2}=0 \text { on } \partial V_{2} .
$$

(d) Complementary variational principles: From the results in (b) and (c) we have the global complementary variational principles

$$
G\left(\Phi_{2}\right) \leq I(\phi) \leq J\left(\Phi_{1}\right)
$$

when

$$
\text { - }(d f / d \psi) \geq 0 \text { for all } \psi,
$$

for all admissible functions $\Phi_{1}$ and $\Phi_{2}$ such that

$$
\begin{array}{rlll}
\sigma_{T} \Phi_{1}=0 & \text { on } & \partial V_{1} \\
\sigma_{T}{ }^{*} T \Phi_{2}=0 & \text { on } & \partial V_{2} .
\end{array}
$$

Equality holds in (3.24) when $\Phi_{1}$ and $\Phi_{2}$ are both equal to the exact function $\phi$.

4. Error bound. For approximate solutions $\Phi_{1}$ which satisfy the exact boundary conditions (2.2) and (2.3), an estimate of the error is available from the error bound [2],

$$
\left\|\Phi_{1}-\phi\right\|_{L^{2}} \leq E\left(\Phi_{1}\right),
$$

where $\|\psi\|_{L^{2}}$ is the usual $L^{2}$ norm $\left[\int_{V} \psi^{2} d V\right]^{1 / 2}$, and

$$
E\left(\Phi_{1}\right)=\left[2 \Lambda^{-1}\left(J\left(\Phi_{1}\right)-I^{-}\right)\right]^{1 / 2} .
$$

Here $I^{-}$denotes a lower bound for $I(\Phi)$, and $\Lambda$ is a lower bound to the lowest eigenvalue of the problem

$$
L \psi=\lambda \psi \quad \text { in } V
$$


with

$$
\begin{array}{rll}
\sigma_{T} \psi=0 & \text { on } & \partial V_{1} \\
\sigma_{T} * T \psi=0 & \text { on } & \partial V_{2}
\end{array}
$$

5. Integral approach. Hereafter, we take $L$ to be a differential operator satisfying the required conditions and suppose that the sufficient conditions for complementary variational principles are satisfied. Let the Green's operator corresponding to $L$ and its domain of functions be $K$, an integral operator with kernel $\mathcal{K}(\mathbf{r}, \mathbf{s})$. Then formally we have

$$
K=L^{-1}
$$

and Eqs. (2.1) to (2.3) may be rewritten as

$$
\phi=K\{f(\phi)\} \quad \text { in } V
$$

where the boundary conditions have been absorbed into the operator $K$. Setting

$$
\theta=f(\phi)
$$

and

$$
m(\theta)=f^{-1}(\theta)=\phi,
$$

we can write Eq. (5.2) as

$$
K \theta=m(\theta) \text { in } V
$$

and since the boundary conditions have been absorbed we take

$$
\sigma_{T}=\sigma_{T}^{*}=0 \text { on } \partial V_{1} \text { and } \partial V_{2} \text {. }
$$

It is shown in [4] that provided the sufficient condition (3.25) is satisfied and certain constants of integration are suitably chosen we obtain the complementary bounds

$$
-\tilde{J}\left(\theta_{1}\right) \leq I(\phi) \leq-\widetilde{G}\left(\theta_{2}\right)
$$

where

$$
\begin{aligned}
& \tilde{J}\left(\Theta_{1}\right)=\int_{V}\left\{\frac{1}{2} \Theta_{1} K \Theta_{1}-M\left(\Theta_{1}\right)\right\} d V, \\
& \widetilde{G}\left(\Theta_{2}\right)=\int_{V}\left\{\left(K \Theta_{2}\right)\left[m^{-1}\left(K \Theta_{2}\right)-\frac{1}{2} \Theta_{2}\right]-M\left[m^{-1}\left(K \Theta_{2}\right)\right]\right\} d V, \\
& M(\theta)=\int^{\theta} m(\psi) d \psi,
\end{aligned}
$$

and $\theta_{1}, \theta_{2}$ are approximations to the exact solution $\theta$ of Eq. (5.5).

6. Problem I: horizontal cantilever with a vertical point load at the free end. By introducing the dimensionless variable $t=s / L$, where $s$ is the distance along the beam, the boundary-value problem satisfied by the angle $\phi$, measured between the tangent 
at a point on the beam, and the horizontal, is as follows:

$$
\begin{aligned}
-d^{2} \phi / d t^{2} & =\left(P L^{2} / E I\right) \cos \phi, \quad 0 \leq t \leq 1, \\
\phi(0) & =0, \quad(d \phi / d t)(1)=0 .
\end{aligned}
$$

The cantilever is assumed to be inextensible, of length $L$ and flexural rigidity $E I$. It is fixed at the end $t=0$, and subjected to a vertical point load $P$ at the free end $t=1$.

7. Complementary variational principles for Problem I. This problem corresponds to the following choices in the general theory:

$$
\begin{aligned}
V & =(0,1), \quad \partial V_{1}=\{t=0\}, \quad \partial V_{2}=\{t=1\}, \\
L & =d^{2} / d t^{2}, \\
T & =d / d t, \quad T^{*}=-d / d t, \quad \sigma_{T}=+1 \quad \text { at } \quad t=1, \quad-1 \quad \text { at } \quad t=0 \\
f(\phi) & =q^{2} \cos \phi,
\end{aligned}
$$

where $q^{2}=P L^{2} / E I$. Then we take

$$
F(\phi)=q^{2} \sin \phi
$$

and the Green's operator $\Re$ is found to have kernel

$$
\Re(t, z)=\min (t, z) \quad 0 \leq t, \quad z \leq 1 .
$$

From (7.4)

$$
-(d f / d \psi)(\psi)=q^{2} \sin \psi
$$

which is nonnegative since $0 \leq \psi \leq \pi / 2$ on physical grounds, and therefore the previous theory may be applied to obtain global complementary variational principles.

For the various functionals we find

$$
\begin{aligned}
I(\phi) & =\int_{0}^{1} q^{2}\left\{\frac{1}{2} \phi \cos \phi-\sin \phi\right\} d t \\
J\left(\Phi_{1}\right) & =\int_{0}^{1}\left\{\frac{1}{2}\left(\frac{d \Phi_{1}}{d t}\right)^{2}-q^{2} \sin \Phi_{1}\right\} d t \text { with } \Phi_{1}(0)=0 \\
G\left(\Phi_{2}\right) & =\int_{0}^{1}\left\{-\frac{d^{2} \Phi_{2}}{d t^{2}} \cos ^{-1}\left[-\frac{1}{q^{2}} \frac{d^{2} \Phi_{2}}{d t^{2}}\right]-\frac{1}{2}\left(\frac{d \Phi_{2}}{d t}\right)^{2}\right. \\
& \left.-q^{2} \sin \left(\cos ^{-1}\left[-\frac{1}{q^{2}} \frac{d^{2} \Phi_{2}}{d t^{2}}\right]\right)\right\} d t \text { with } \frac{d \Phi_{2}}{d t}(1)=0 \\
\tilde{J}\left(\Theta_{1}\right) & =\int_{0}^{1}\left\{\frac{1}{2} \Theta_{1} K \Theta_{1}-\Theta_{1} \cos ^{-1}\left[\frac{\Theta_{1}}{q^{2}}\right]+q^{2}\left[1-\left(\frac{\Theta_{1}}{q^{2}}\right)^{2}\right]^{1 / 2}\right\} d t \\
\tilde{G}\left(\Theta_{2}\right) & =\int_{0}^{1}\left\{-\frac{1}{2} \Theta_{2} K \Theta_{2}+q^{2} \sin \left(K \Theta_{2}\right)\right\} d t .
\end{aligned}
$$

Calculations have been performed for the case $q^{2}=2$. Simple polynomials in $t$ were taken as the trial functions:

$$
\Phi_{1}=\sum_{r=1}^{6} a_{r} t^{r},
$$


TABLE I. Differential approach variational parameters for problem I.

\begin{tabular}{ccccccc}
\hline$a_{1}$ & $a_{2}$ & $a_{3}$ & $a_{4}$ & $a_{5}$ & $a_{6}$ & $J\left(\Phi_{1}\right)$ \\
\hline 1.67868 & $-9.99995(-1)$ & $4.42960(-3)$ & $2.17196(-1)$ & $-1.50552(-1)$ & $3.19995(-2)$ & -0.56478528 \\
\hline$b_{1}$ & $b_{2}$ & $b_{3}$ & $b_{4}$ & $b_{5}$ & $b_{6}$ & $G\left(\Phi_{2}\right)$ \\
\hline 1.67868 & $-9.99995(-1)$ & $4.39960(-3)$ & $2.17196(-1)$ & $-1.50542(-1)$ & $3.20060(-2)$ & -0.56478564 \\
\hline
\end{tabular}

Here $m(-n)$ means $m \times 10^{-n}$.

$$
\begin{array}{ll}
\Phi_{2}=\sum_{r=1}^{6} b_{r} t^{r}, & \\
\Phi_{3}=\sum_{r=1}^{3} c_{r} t^{r}, & \Theta_{1}=q^{2} \cos \Phi_{3}, \\
\Phi_{4}=\sum_{r=1}^{3} d_{r} t^{r}, & \theta_{2}=q^{2} \cos \Phi_{4},
\end{array}
$$

the boundary conditions in (6.2) were imposed on all the trial functions $\Phi_{1}$ to $\Phi_{4}$, and then the functionals were optimized over the remaining free parameters. To estimate the error in $\Phi_{1}$ from (4.2) we require $\Lambda$ which, on using Eqs. (7.1) to (7.3), was found to be

$$
\Lambda=\pi^{2} / 4 \text {. }
$$

The optimum functional and parameter values are given in Tables I and II, and from these we see that $-\widetilde{J}\left(\theta_{1}\right)$ produced a better lower bound for $I(\phi)$ than $G\left(\Phi_{2}\right)$, using fewer parameters. This value was substituted for $I^{-}$in the error estimate, giving

$$
E\left(\Phi_{1}\right)=3.37 \times 10^{-4},
$$

indicating that the trial function $\Phi_{1}$ is quite accurate. This is borne out by calculating the vertical and horizontal displacements by numerical integration of $\sin \Phi_{1}$ and $\cos \Phi_{1}$ and comparing these with the corresponding values found from an approach given in Frisch-Fay [5], based upon complete and incomplete elliptic integrals. The comparisons are given in Table III.

TABLE II. Integral approach variational parameters for Problem I.

\begin{tabular}{cccc}
\hline$c_{1}$ & $c_{2}$ & $c_{3}$ & $-J\left(\theta_{1}\right)$ \\
\hline 1.67030 & $-9.96970(-1)$ & $1.07880(-1)$ & -0.56478541 \\
\hline$d_{1}$ & $d_{2}$ & $d_{3}$ & $-\tilde{G}\left(\theta_{2}\right)$ \\
\hline 1.65152 & $-9.54492(-1)$ & $8.58211(-2)$ & -0.56478522 \\
\hline
\end{tabular}

TABLE III. Comparison of the coordinates of the deflected shape for Problem I.

\begin{tabular}{lcccc}
\hline \multicolumn{1}{c}{$t$} & $x / L$ using $\Phi_{1}$ & $x / L$ from $[5]$ & $y / L$ using $\Phi_{1}$ & $y / L$ from $[5]$ \\
\hline 0.25 & 0.244229 & 0.244230 & 0.046764 & 0.046764 \\
0.5 & 0.464534 & 0.464536 & 0.163650 & 0.163647 \\
0.75 & 0.659356 & 0.659358 & 0.319999 & 0.319996 \\
1.0 & 0.839355 & 0.839358 & 0.493462 & 0.493458 \\
\hline
\end{tabular}


8. Problem II: horizontal cantilever with uniformly distributed load. It is not possible to use the elliptic integral theory for this problem and recourse to techniques supplying an accurate numerical approximation must be made.

The same assumptions hold as in Problem I, but the vertical point load is replaced by uniformly distributed load per unit length $u$, and the free end occurs at $t=0$, the fixed end at $t=1$. In this case the nonlinear boundary-value problem takes the form [6]:

$$
\begin{aligned}
-d^{2} \phi / d t^{2} & =\left(w L^{3} / E I\right) t \cos \phi, \quad 0 \leq t \leq 1 ; \\
d \phi / d t(0) & =0, \quad \phi(1)=0 .
\end{aligned}
$$

9. Complementary variational principles for Problem II. We have the following cases of the basic theory:

$$
\begin{aligned}
& V=(0,1), \quad \partial V_{1}=\{t=1\}, \quad \partial V_{2}=\{t=0\}, \\
& L=-d^{2} / d t^{2}, \\
& T=d / d t, \quad T^{*}=-d / d t, \quad \sigma_{T}=+1 \quad \text { at } t=1, \quad-1 \quad \text { at } t=0, \\
& f(\phi)=r^{2} t \cos \phi,
\end{aligned}
$$

where $r^{2}=w L^{3} / E I$. We take

$$
F(\phi)=r^{2} t \sin \phi
$$

and the Green's operator $K$ has kernel

$$
\Re(t, z)=1-\max (t, z), \quad 0 \leq t, \quad z \leq 1 .
$$

From Eq. (9.4)

$$
-(d f / d \psi)(\psi)=r^{2} t \sin \psi,
$$

which again is nonnegative on physical grounds, and the global complementary variational principles hold.

The functionals take the forms

$$
\begin{aligned}
I(\phi) & =\int_{0}^{1} r^{2} t\left\{\frac{1}{2} \phi \cos \phi-\sin \phi\right\} d t \\
J\left(\Phi_{1}\right) & =\int_{0}^{1}\left\{\frac{1}{2}\left(\frac{d \Phi_{1}}{d t}\right)^{2}-r^{2} t \sin \Phi_{1}\right\} d t \quad \text { with } \Phi_{1}(1)=0 \\
G\left(\Phi_{2}\right) & =\int_{0}^{1}\left\{-\frac{d^{2} \Phi_{2}}{d t^{2}} \cos ^{-1}\left[-\frac{1}{\left(r^{2} t\right)} \frac{d^{2} \Phi_{2}}{d t^{2}}\right]-\frac{1}{2}\left(\frac{d \Phi_{2}}{d t}\right)^{2}\right. \\
& \left.-r^{2} t \sin \left(\cos ^{-1}\left[-\frac{1}{\left(r^{2} t\right)} \frac{d^{2} \Phi_{2}}{d t^{2}}\right]\right)\right\} d t \text { with } \frac{d \Phi_{2}}{d t}(0)=0 \\
\widetilde{J}\left(\Theta_{1}\right) & =\int_{0}^{1}\left\{\frac{1}{2} \Theta_{1} K \Theta_{1}-\Theta_{1} \cos ^{-1}\left[\frac{\Theta_{1}}{r^{2}}\right]+r^{2} t\left[1-\left(\frac{\Theta_{1}}{r^{2}}\right)^{2}\right]^{1 / 2}\right\} d t \\
\tilde{G}\left(\Theta_{2}\right) & =\int_{0}^{1}\left\{-\frac{1}{2} \Theta_{2} K \Theta_{2}+r^{2} t \sin \left(K \Theta_{2}\right)\right\} d t .
\end{aligned}
$$


TABLE IV. Differential approach variational parameters for Problem II.

\begin{tabular}{ccccc}
\hline$a_{0}$ & $a_{3}$ & $a_{4}$ & $a_{5}$ & $J\left(\Phi_{1}\right)$ \\
\hline $3.21552(-1)$ & $-3.14132(-1)$ & $-2.35000(-3)$ & $-5.07000(-3)$ & -0.097848772 \\
\hline$b_{0}$ & $b_{3}$ & $b_{4}$ & $b_{5}$ & $G\left(\Phi_{2}\right)$ \\
\hline $3.21880(-1)$ & $-3.10680(-1)$ & $-1.10100(-2)$ & $-1.90000(-4)$ & -0.097878123 \\
\hline
\end{tabular}

Calculations have been performed for the case $r^{2}=2$. Again simple polynomials in $t$ were used for trial functions:

$$
\begin{aligned}
& \Phi_{1}=\sum_{r=0}^{5} a_{r} t^{r}, \\
& \Phi_{2}=\sum_{r=0}^{5} b_{r} t^{r}, \\
& \Phi_{3}=\sum_{r=0}^{3} c_{r} t^{r}, \quad \Theta_{1}=r^{2} t \cos \Phi_{3}, \\
& \Phi_{4}=\sum_{r=0}^{3} d_{r} t^{r}, \quad \theta_{2}=r^{2} t \cos \Phi_{4},
\end{aligned}
$$

where the prime on the summation denotes that we omit the terms $t$ and $t^{2}$. The former are omitted in order that $\Phi_{1}-\Phi_{4}$ satisfy the first boundary condition in (8.2), the latter omitted to avoid the term $-\left(d^{2} \Phi_{2} / d t^{2}\right) /\left(r^{2} t\right)$ in $G\left(\Phi_{2}\right)$ becoming infinite at $t=0$. After imposing the second boundary condition of $(8.2)$ on $\Phi_{1}-\Phi_{4}$, the functionals were optimized over the remaining free parameters. The value of $\Lambda$ is the same as in Problem I, as given in Eq. (7.17), and the optimum functional and parameter values for this problem are given in tables IV and V. Again $-\widetilde{J}\left(\theta_{1}\right)$ produced the better lower bound of $I(\phi)$ and on substitution for $I^{-}$in the error bound we obtain

$$
E\left(\Phi_{1}\right)=1.76 \times 10^{-4} .
$$

The vertical and horizontal displacements for points along the beam were calculated as in Problem I and compared with those from a power series approximation used by Rohde [6]. The comparisons are given in Table VI. Given the values of $E I, L$ and $w$, this seems to be a more logical approach to the problem.

TABLE V. Integral approach variational parameters for Problem II.

\begin{tabular}{ccc}
\hline$c_{0}$ & $c_{3}$ & $-\widetilde{J}\left(\theta_{1}\right)$ \\
\hline $3.22300(-1)$ & $-3.22300(-1)$ & -0.097848809 \\
\hline$d_{0}$ & $d_{3}$ & $-\tilde{G}\left(\theta_{2}\right)$ \\
\hline $3.22300(-1)$ & $-3.22300(-1)$ & -0.097848780 \\
\hline
\end{tabular}


TABLE VI. Comparison of the coordinates of the deflected shape for Problem II.

\begin{tabular}{lcccc}
\hline \multicolumn{1}{c}{$t$} & $x / L$ using $\Phi_{1}$ & $x / L$ from $[6]$ & $y / L$ using $\Phi_{1}$ & $y / L$ from $[6]$ \\
\hline 0.25 & 0.237283 & 0.237280 & 0.078718 & 0.078727 \\
0.5 & 0.475880 & 0.475877 & 0.153319 & 0.153327 \\
0.75 & 0.718572 & 0.718570 & 0.212931 & 0.212937 \\
1.0 & 0.966895 & 0.966893 & 0.238500 & 0.238507 \\
\hline
\end{tabular}

\section{REFERENCES}

[1] A. M. Arthurs, Complementary variational principles, Clarendon Press, Oxford, 1970

[2] A. M. Arthurs, Dual extremum principles and error bounds for a class of boundary value problems, J. Math. Anal. Appl. 41, 781-795 (1973)

[3] S. G. Mikhlin, Variational methods in mathematical physics, Pergamon Press, Oxford, 1964, p. 350

[4] N. Anderson, A. M. Arthurs and P. D. Robinson, Pairs of complementary variational principles, J. Inst. Maths. Applics. 5, 422-431 (1969)

[5] R. Frisch-Fay, Flexible bars, Butterworths, London, 1962, p. 35

[6] F. V. Rohde, Large deflections of a cantilever beam with uniformly distributed load, Quart. Appl. Math. $11,337-338(1953)$ 